mating. Second, females may prefer male characteristics that are indicators of the genetic marker rather than the marker itself. It is important to uncover the phenotype that females use to choose mates as well as the genetic qualities it indicates.

ANDREW POMIANKOWSKI Department of Genetics and Biometry, University College London, London NW1 2HE, UK

Department of Mathematics, MARK PAgEL Queen Mary's College, London E1 4NF, UK

PotTs ET AL. REPLY - Pomiankowski and Pagel raise some important issues which space did not permit us to address in our paper ${ }^{1}$. First, it is important to establish that of the three kinds of mating preferences that could account for our observed deficiency of MHC homozygous progeny - preferences for heterozygotes, for rarity or for disparity - our data support only the last of these. A mating advantage for males with a rare MHC can be excluded because this hypothesis predicts an excess of rare homozygotes, whereas a deficiency is observed (in preparation).

A mating advantage for MHCheterozygous males would, as Pomiankowski and Pagel suggest, result in a deficiency of MHC-homozygous offspring, but only if populations are in Hardy-Weinberg equilibrium; ours were not. They were founded with equal numbers of MHC heterozygotes and homozygotes to enhance tests of the overdominance hypothesis. So, even under the extreme and demonstrably false (see below) assumption that females reject all MHC-homozygous males, the expected number of $\mathrm{MHC}$ homozygous offspring does not differ significantly from random mating expectations. Consequently, a mating advantage for MHC-heterozygous males could not explain our observed deficiency of homozygous progeny.

In addition, the following data directly test the examples cited by Pomiankowski and Pagel. First, the mean territory size for MHC-heterozygotes $\left(8.8 \mathrm{~m}^{2}\right)$ does not differ from homozygotes $\left(9.6 \mathrm{~m}^{2}\right)$, so random encounter rates by females would accurately reflect true genotypic proportions of territorial males. In fact, random extraterritorial matings would have produced fewer MHC-heterozygous offspring due to the nonrandom settlement patterns favouring MHCdisparate pairs ${ }^{1}$. Second, for the extraterritorially mating females in Table 3 of ref. 1, mating was random with respect to MHC homozygosity or heterozygosity of males $\left(\chi^{2}=0.05, P=0.82\right)$. But these matings showed an MHC- disassortative pattern which departed significantly from the random expectations proposed by Pomiankowski and Pagel $\left(\chi^{2}=5.02, P=0.025\right)$.

Pomiankowski and Pagel are concerned that the reason for $\mathrm{MHC}$ disassortative mate choice is undercut if MHC heterozygotes are not fitter. In general, we agree with this concern, but it is possible that MHC heterozygotes enjoy a fitness advantage too weak to be detected with the sample size of our study $^{2}$. For example, a selection coefficient of $s=0.01$ operating against homozygotes would be undetectable in our system, but would be a strong evolutionary force favouring MHC-based mating preferences ${ }^{2}$. Alternatively, our manipulation of genome-wide inbreeding (Table 1 of ref. 1) resulted in detectable levels of inbreeding depression. Thus, fitness consequences of MHC-based inbreeding avoidance may still be detectable in experiments similar to ours.

Finally, Pomiankowski and Pagel raise the problem of distinguishing preferences for correlated characters from characters directly dependent on MHC. This is a general problem when a single class of mates is preferred, but under disassortative mating every class of prospective mates are preferred by some class of choosers. For example, a preference for vigour could favour heterozygotes or rare males, but it could not lead to disassortative patterns because such preferences depend on the chooser's genotype.

Correlated characters arising from linked genes offer one remaining problem, which has been addressed by numerous genetically controlled experiments demonstrating that classical $\mathrm{MHC}$ loci are dominant in controlling individual odours ${ }^{3}$ and in influencing laboratory mating preferences ${ }^{3,4}$. These experiments make use of $\mathrm{F}_{2} \mathrm{~s}$ from congenic strains, specific mutations within the $\mathrm{MHC}$, cross-fostering (reviewed in ref. 3 ) and germ-free mice 5 . Combining these results with the considerations discussed above makes it unlikely that MHC-based mating preferences result from either correlated characters or genes other than MHC loci themselves.

WAYNE K. POTTS

C. JO. MANNING*

EDWARD K. WAKELAND

Center for Mammalian Genetics,

University of Florida,

Gainesville, Florida 32610, USA

*Also at: Department of Psychology, University of Washing ton, Seattle, Washington 98195, USA.

1. Potts, W. K., Manning, C. J. \& Wakeland, E. K. Nature 352, 619-621 (1991)

2. Potts, W. K. \& Wakeland, E. K. Trends ecol. Evol. 5 181-187 (1990)

3. Boyse, E. A. et al. in Psychoneuroimmunology 2 nd edn (eds Ader, R et al) 831 (Academic, New York, 1990).

4. Egid. K. \& Brown, J. L. Anim. Behav. 8, 548-549 (1989).

5. Yamazaki, K. et al. Proc. natn. Acad. Sci. U.S.A. 87. 5. Yamazaki, K. et al.
9413-8416 (1990).

\section{Sustainable populations?}

SIR - Hoppe's provocative assertion that "Brazil has no population pressure . . ." (Nature 355, 593-594; 1992) begs the question of how overpopulation can be recognized. Subjectively chosen indicators might perhaps include nonsustainable internal consumption of resources, mass starvation or unemployment.

Ecologists, economists and politicians might have different opinions as to whether an area is, or will become, overpopulated. I trust that most geologists, and Hoppe, could recognize population pressure long before the density of people is sufficient to inhibit their mining projects!

The crucial but controversial issue of the sustainable population of individual countries, and of the globe, demands international and interdisciplinary agreement; the forthcoming environmental summit in Brazil should be a suitable venue for discussion.

\section{Department of Zoology,}

University of Oxford, Oxford OX1 3PS, UK

SIR - Hoppe ${ }^{1}$ is wrong in his view that Amazonian rain forests cannot be exploited sustainably. Tropical rain forests, just like others, are dynamic ecosystems ${ }^{2}$. Trees are continuously dying individually or in groups to create canopy gaps that are the first stage of a growth cycle. Gaps are colonized by seedlings that grow up eventually to form a new mature forest. This is well known to foresters who have developed silvicultural systems that work within the inherent biological limits and allow the harvesting of trees as they mature.

Such systems are well established in the Asian rain forests ${ }^{3}$ and have recently been devised for the neotropics ${ }^{4,5}$. Economic exploitation is sustainable so long as it is carefully conducted to minimize erosion, soil compaction and damage to juvenile trees that will form the next growth cycle. Careless or excessively heavy exploitation delays recovery but does not destroy forest. What does destroy forest is its conversion to either crops or pastures. For timber extraction roads are built and often destruction occurs because these give access to

1. Hoppe, E. Nature 355, 593-594 (1992).

. Whitmore, T. C. Introduction to Tropical Rain Forests (Clarendon, Oxford, 1990).

3. Wyatt-Smith, J. Malayan Forest Records 23, 1963.

4. de Graaf, N. R. A Silvicultural System for Natural Regeneration of Tropical Rain Forest in Suriname (Agricultural University, Wageningen, 1986).

5. Silva, J. N. M. The Behaviour of the Tropical Rain Forest of the Brazilian Amazon after Logging thesis (University of Oxford, 1989) 